\title{
Les crues d'automne 1958 sur le Vidourle
}

\section{The Autumn 1958 floods on the Vidourle}

\author{
PAR .J. . TACQUET
}

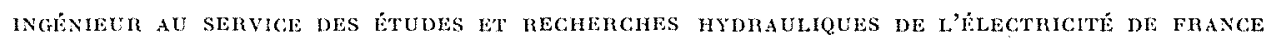

\begin{abstract}
Analyse de la formation et de la propagation des crues du Vidourle. - Observations des crues de 1958 (plaviographes et limnigraphes). Causes météorologiques de lear violence. - Influence sur leur évolution de la position du centre de gravité des averses génératrices. Comparaison des crues de 1958 ana records du passé (niveaux atteints et débits spécifiques maxima). - Recherche d'une relation pluiedébit pour le calcul des débits de pointe par la methode de linydrogramme unitaire. - Etude des temps de concentration et des coofficients de ruissellement. - Interet des hydrogrammes unitaires pour les cours d'eou de type cévenol.
\end{abstract}

\begin{abstract}
Analysis of the formation and propagation of floods on the Vidourle.-Flood observations in 1958 (rain and level recording ganges).-Meteorological reasons for the biolence of the floods. The effect on the floods of the position of the centroids of the storms lhat caused them. Comparison of the 1958 floods with record floods in the past (stages and peak flows).Setting up a precipilation-discharge relation for the determination of peak flows by the unit hydrograph method.-Investigation of times of concentration and ranoff percentages.-The advantages of applying the unit hydrograph method to watercourses of the Cevennes type.
\end{abstract}

\section{INTRODUCTION}

Le Vidourle, petit fleuve côtier de la Méditerranée, draine un bassin d'environ $800 \mathrm{~km}^{2}$ depuis sa source à la montagne de la Fage (S.E. du massif de l'Aigoual) jusqu'au pont romain de Grand-Gallargues à partir duquel son cours, endigué jusqu'à la mer, traverse la plaine du Bas-Languedoc.

Comparé à ses voisins, Gard et Héraull, le Vidourle $(90 \mathrm{~km}$ de longueur y compris les $22 \mathrm{~km}$ de chenal endigué à l'aval de Grand-Gallargues) est un cours d'eau modeste. Néanmoins, la protection des localités riveraines (Saint-Hippolytedu-Fort, Sauve, Quissac et Sommières) contre ses violentes et relativement fréquentes crues d'automne et les ruptures de digues qu'elles occasionnent dans la plaine côtière, constituent des problèmes importants et anciens; au xviri s. Pitot s'y intéressa.

Pour assurer cette protection, la construction de barrages écréteurs de crues est envisagée sur le cours supérieur du Vidourle et de certains de ses affluents (Rieumassel et Crieulon).

Depuis 1956, fonctionne sur le bassin, pour l'étude de ces ouvrages, un réseau relativement dense de stations pluviométriques ( 3 pluviographes, 16 pluviomètres) et limnimétriques : 4 limnigraphes, une dizaine d'échelles limnimétriques régulièrement obşervées et une station de jaugeage permanente du type téléférique installée à Sommières (cf. carte $n^{\circ} 1$ ). Cet équipement a permis d'observer assez complètement les crues des $1^{\text {er }}$ et 4 octobre 1958 qui sont particulièrement caractéristiques du Vidourle.

La présente communication concerne essentiellement le régime des crues du Vidourle et de ses affluents à l'amont de Sommières, localité la plus importante du bassin, où les submersions sont fréquentes (1 année sur deux en moyenne) et particulièrement dommageables (plusieurs centaines de millions de francs de dégâts le 4 octobre 1958). 


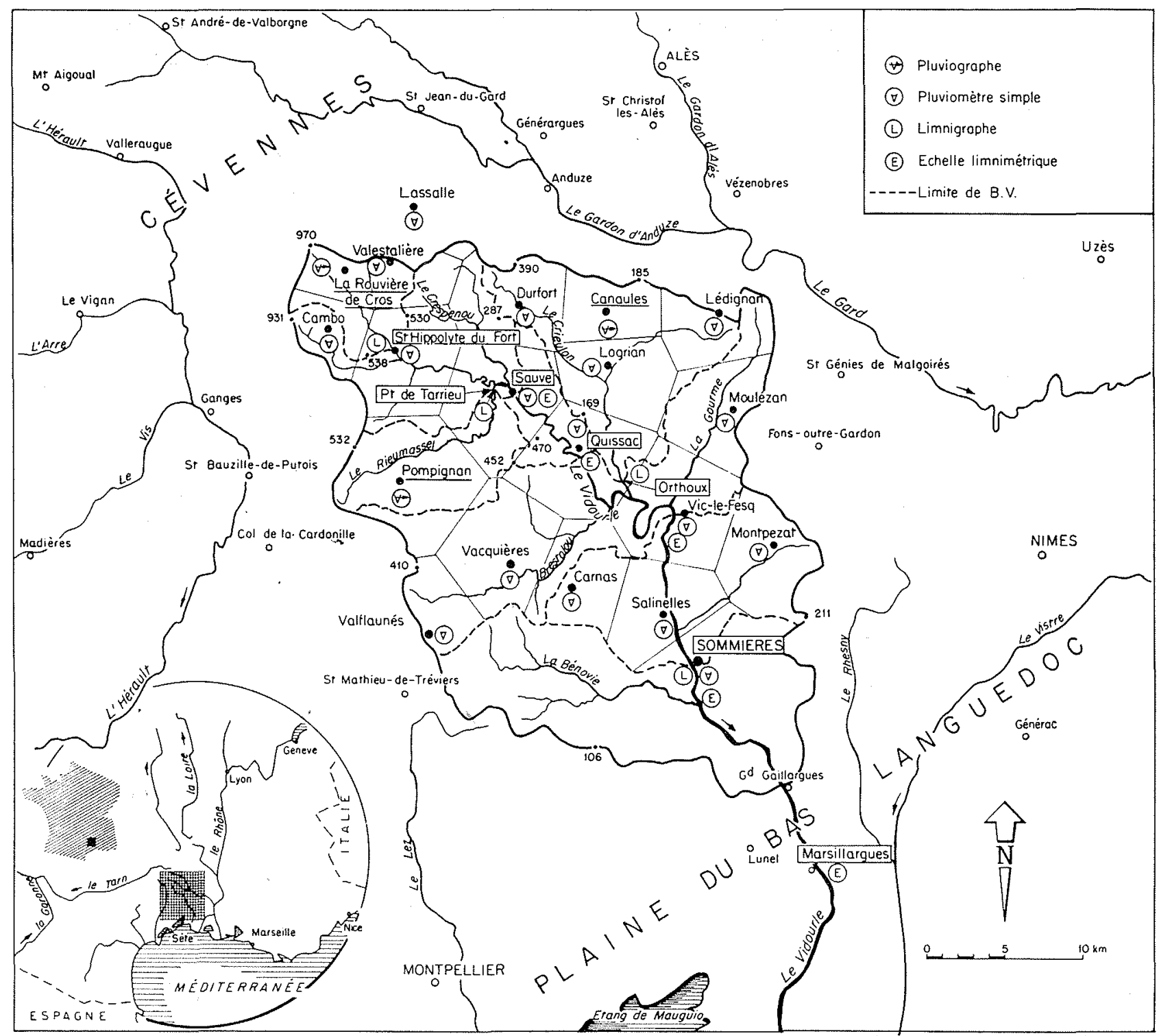

Carte $\mathrm{N}^{\circ}$ 1. - Equipement pluviométrique et limnimétrique du bassin versant du vidourle.

\section{A. - L'INFRASTRUCTURE DU BASSIN CONSIDÉRÉE DU POINT DE VUE DE LA FORMATION ET DE LA PROPAGATION DES CRUES}

\section{I. - Le relief et le réseau hydrographique}

Le relief est concentré sur le quart nord-ouest du bassin. Bien qu'aucun point n'atteigne $1000 \mathrm{~m}$ d'altitude (la montagne du Liron culmine à $975 \mathrm{~m}$ ), le modelé des massifs qui le constituent est très accusé : le Vidourle à l'amont de SaintHippolyte-du-Fort présente toutes les caractéristiques d'un torrent cévenol dont la pente dépasse $60 / 1000$.
A l'aval de Saint-Hippolyte-du-Fort, la concentration d'ondes de crues est favorisée par les lignes de plissement qui compartimentent le bassin : l'effet de la décroissance de pente est ainsi aggravé, du point de vue de l'écoulement des crues, par des étranglements au franchissement de ces lignes et par le débouché de couples d'affluents importants à même hauteur sur le Vidourle (Rieumassel et Crespenou à Sauve, Brestalou et Crieulon à l'aval de Quissac). 


\section{II. - La nature géologique des terrains et ses incidences hydrologiques}

La proportion des sols «imperméables» est voisine de $65 \%$ de la superficie des bassins versants lorsqu'on se déplace de l'amont à l'aval, les $35 \%$ restants étant constitués de terrains «perméables en grand» (calcaires fissurés et karst jurassiques principalement à l'amont de Sauve), et « relativement perméables », qui prennent une certaine extension à l'aval de Quissac.

Nous insisterons particulièrement ici sur les phénomènes qui affectent le Vidourle et le Rieumassel dans la traversée des zones larstiques «perméables en grand» qui s'étendent de l'Hérault au Gardon d'Anduze.

a) Les pertes de débit du Vidourle entre SaintHippolyte et Sauve sont telles que son lit superficiel est habituellement à sec entre ces deux localités. Son lit souterrain, exploré par Martel, de Joly, etc., débouche à la résurgence de Sauve. Quant au Rieumassel; qui draine la cuvette de Pompignan et dont le débit apparent est également nul en temps normal, son lit souterrain, mal connu, rejoint celui du Vidourle près de Sauve.

b) La capacité du réseau hydrographique souterrain ainsi constitué dans le karst permet dans certains cas $d$ ' «encaisser » une crue du Vidourle supérieur qui, relativement importante à Saint-Hippolyte, passe inaperçue à l'aval (par exemple, les 20 et 21-9-1957 où une crue de $700 \mathrm{l} / \mathrm{s} / \mathrm{km}^{2}$ à Saint-Hippolyte a été ainsi neutralisée). En fait, la perméabilité en grand des massifs karstiques vient le plus souvent aggraver l'effet des terrains imperméables $\left(^{*}\right)$ sur la formation d'ondes de crues brutales à l'amont de Sauve. Lors des averses intenses d'automne, une partie des précipitations ruisselle à travers la masse du karst et des volumes d'eau considérables s'accumulent ainsi rapidement dans les avens qui jalonnent le réseau hydrographique souterrain.

Dans cette zone, seuls les débits qui excèdent la capacité instantanée d'absorption du réseau souterrain transitent par le lit superficiel des cours d'eau. Correspondant aux apports de la phase de ruissellement superficiel des averses, ils constituent des ondes de crue à front raide qui se propagent à la façon d'un mascaret, le phénomène étant amplifié par l'entrée en action soudaine d'évents ou d'exsurgences temporaires du réseau souterrain mis en charge. Il en résulte les montées d'eau et vitesses de propagation extrêmement rapides observées à l'amont de Sauve, point du bassin où la concentration des

(*) Ces deux natures de terrains constituent $95 \%$ de la superficie du bassin versant du vidourle à Sauve et $100 \%$ dans le cas du Rieumassel. crues atteint son paroxysme (cf. les enregistrements de crues du Rieumassel au pont de Tarrieu).

\section{III. - La couverture végétale}

La couverture végétale $d u$ bassin n'est pas susceptible de jouer un rôle modérateur important dans les zones de formation des crues du Vidourle: causses et garrigues à végétation clairsemée couvrent $70 \%$ de la superficie du bassin versant à Sauve, et 40 à $45 \%$ de la superficie du bassin total à Grand-Gallargues. Le reboisement du haut-bassin serait peu efficace, en raison de la nature des sols limitant considérablement l'extension de ce boisement et de la violence des averses génératrices de crues.

\section{IV. - Les phénomènes d'érosion et les atterrissements}

L'intensité de ces averses, qui peut dépasser $50 \mathrm{~mm} / \mathrm{h}$ pendant plusieurs heures, explique l'érosion profonde observée sur tous les types de terrains du bassin et, corrélativement, les dépôts de limon dans les champs d'inondation. Des engravements importants dans la traversée des localités nécessitent des travaux fréquents d'entretien du lit (dragage, rectification des berges, etc.) dont l'effet n'est appréciable que sur les crues moyennes: les obstacles majeurs à l'écoulement des crues n'étant généralement pas susceptibles de modifications sensibles.

\section{V. - Les obstacles artificiels à l'écoulement des crues}

Les goulots d'étranglement dangereux pour l'écoulement des crues sont en effet constitués davantage par des aménagements artificiels que par les encaissements naturels du lit mineur : les ponts, les endiguements, les constructions édifiées dans les lits majeur et mineur sont responsables des montées d'eau extraordinaires observées dans les localités riveraines $(9 \mathrm{~m}$ audessus de l'étiage repérés à Sauve, $8,50 \mathrm{~m}$ à Quissac, $7,70 \mathrm{~m}$ à Sommières).

Le cas de Sommières est caractéristique. Cette ville s'est développée autour d'un pont romain qui est aujourd'hui amputé de près de $50 \%$ de sa section d'écoulement. Il comportait primitivement, sur $190 \mathrm{~m}$ de développement, 17 arches offrant à l'écoulement des eaux une section totale de $800 \mathrm{~m}^{2}$. Les quais actuels réduisent le lit du Vidourle à la moitié de son ancienne largeur, soit $90 \mathrm{~m}$ environ et les crues ne disposent pour leur écoulement que de la section des 7 arches majeures du pont $\left(425 \mathrm{~m}^{2}\right.$ au total). Les 10 arches manquantes existent encore enfouies sous l'agglomération ( 7 en rive gauche et 3 en rive droite). Il en résulte que : 


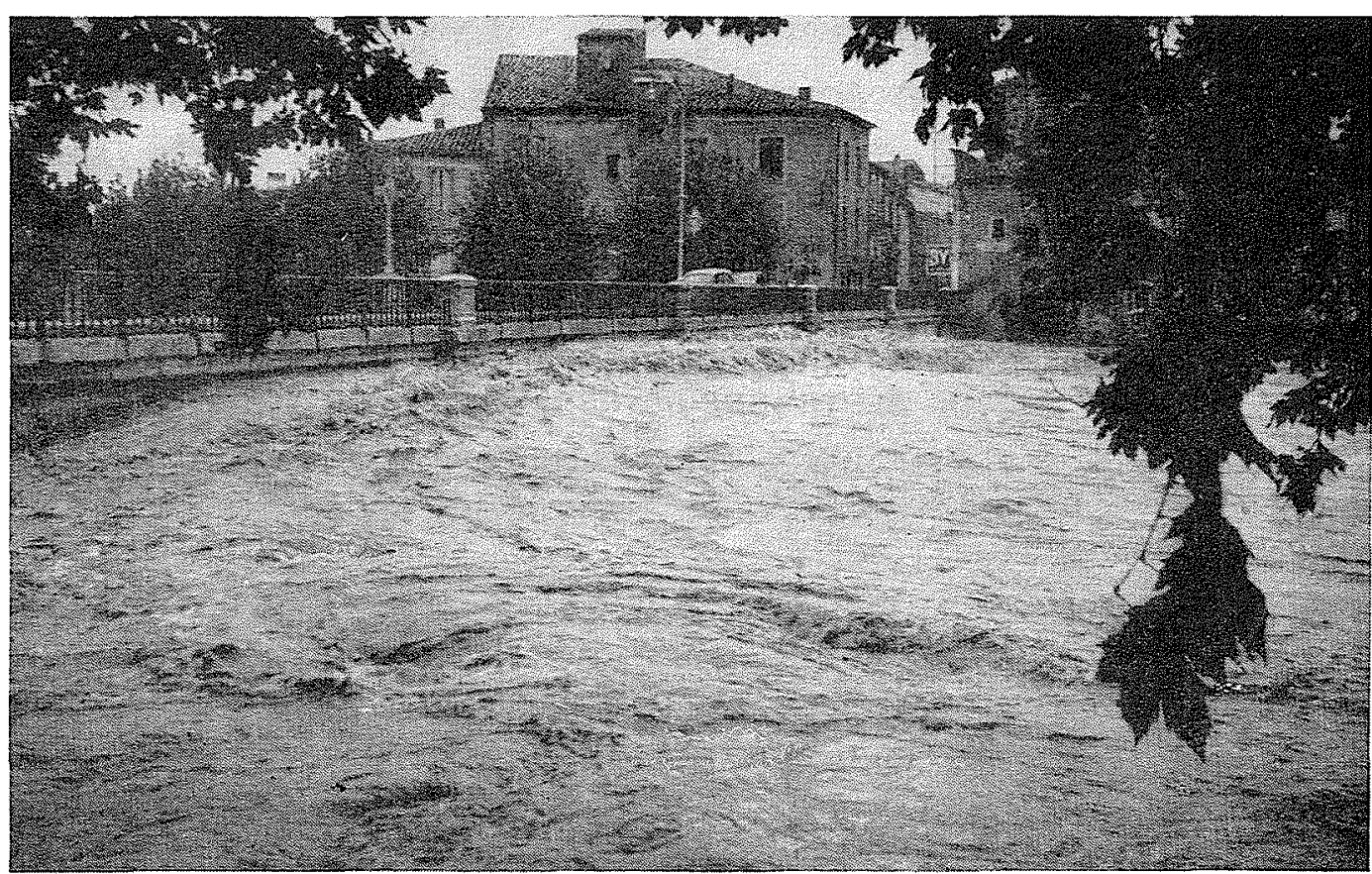

Рното I

Le maximum de la crue du 4 octobre 1958 au pont de Sommières : vue amont.

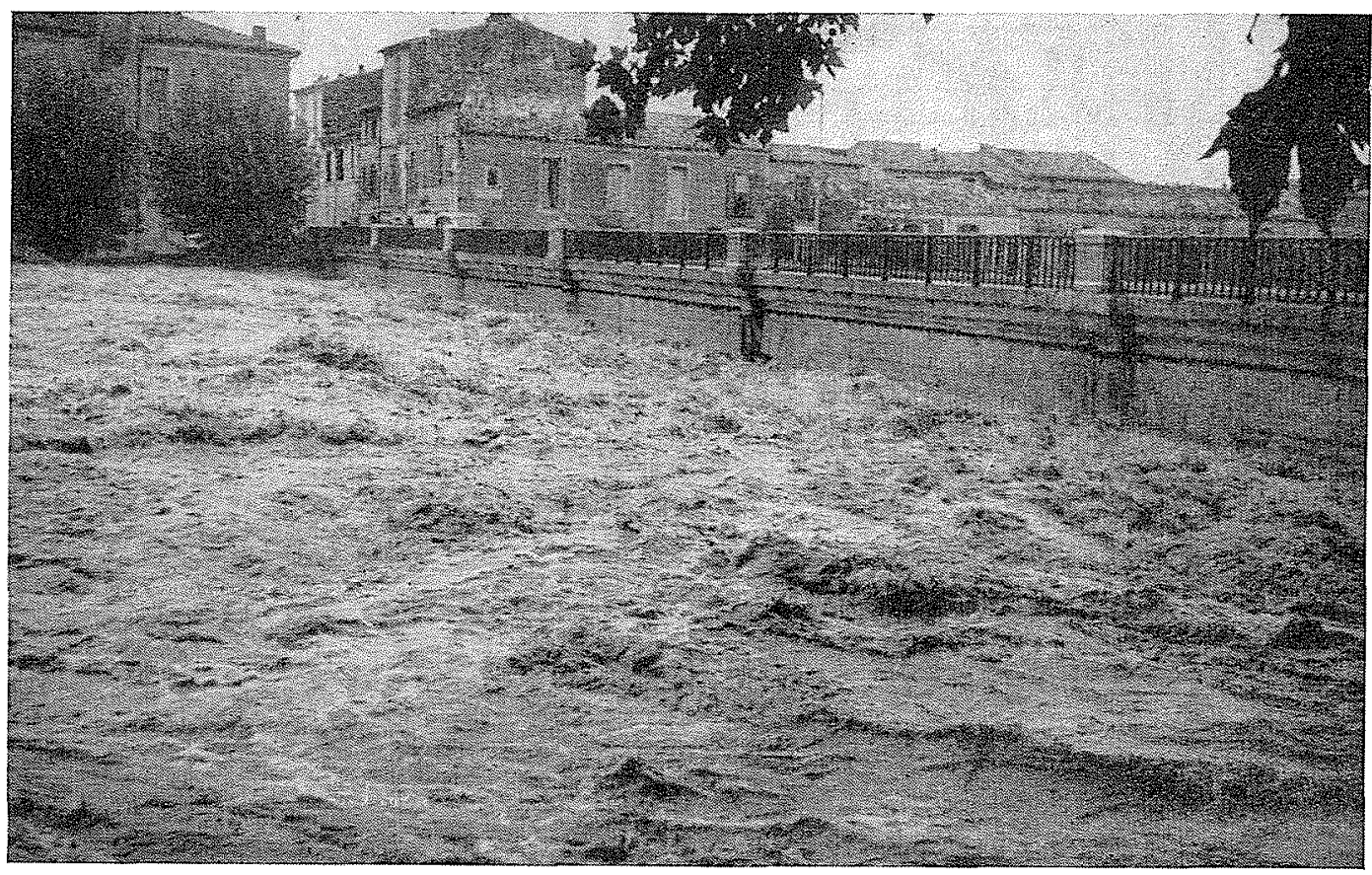

PHoto II

Le maximum de la crue du 4 octobre 1958 au pont de Sommières : vue aval.

a) L'ouvrage a été surmonté à plusieurs reprises $(1723,1933)$ et le phénomène a failli se reproduire le 4 octobre 1958 (Photos I et II).

b) Les bas-quartiers de la ville établis dans le lit majeur sont inondés dès que la cote $3,00 \mathrm{~m}$ (correspondant à un débit de $400 \mathrm{~m}^{3} / \mathrm{s}$ de fréquence biennale) est atteinte et la hauteur des submersions peut $\mathrm{y}$ dépasser $4,50 \mathrm{~m}$ (cas du 4 octobre 1958). 
B. - LES FACTEURS MÉTÉOROLOGIQUES DES CRUES DU VIDOURLE

T A B L E A I

OBSERVATIONS MÉTÉOROLOGIQUES RELATIVES AUX CRUES CÉVENOLES DE SEPTEMBRE-OCTOBRE 1958

A. - PRESSION - TEMPÉRATURE - VENTS

\begin{tabular}{|c|c|c|c|c|c|c|c|c|c|c|c|c|c|}
\hline \multirow{3}{*}{$\begin{array}{c}\text { Date } \\
30-9-58\end{array}$} & \multirow{3}{*}{$\frac{\text { Heure }}{12 \text { h } 00}$} & \multicolumn{4}{|c|}{ Mont Algoval. - Alt. $1567 \mathrm{~m}$} & \multicolumn{4}{|c|}{ Nînes. - Alt. $54 \mathrm{~m}$} & \multicolumn{4}{|c|}{ MONTPELLIER. - Alt. $5 \mathrm{~m}$} \\
\hline & & \multirow{2}{*}{$\begin{array}{c}\begin{array}{c}\text { Pression } \\
(\mathrm{mb}) \\
\cdot\end{array} \\
852,2\end{array}$} & \multirow{2}{*}{$\begin{array}{c}\begin{array}{c}\text { Tempé- } \\
\text { rature } \\
\left({ }^{\circ} \mathrm{C}\right)\end{array} \\
7,7\end{array}$} & \multicolumn{2}{|c|}{$\begin{array}{c}\text { Direction et } \\
\text { vitesse du vent } \\
(\mathrm{m} / \mathrm{s})\end{array}$} & \multirow{2}{*}{$\begin{array}{l}\begin{array}{c}\text { Pression } \\
(\mathrm{mb})\end{array} \\
1004,5\end{array}$} & \multirow{2}{*}{$\begin{array}{c}\begin{array}{c}\text { Tempé- } \\
\text { rature } \\
\text { (" C) }\end{array} \\
22,2\end{array}$} & \multicolumn{2}{|c|}{$\begin{array}{c}\text { Direction et } \\
\text { vitesse du vent } \\
(\mathrm{m} / \mathrm{s})\end{array}$} & \multirow{2}{*}{$\begin{array}{l}\begin{array}{c}\text { Pression } \\
(\mathrm{mb})\end{array} \\
1002,9\end{array}$} & \multirow{2}{*}{$\begin{array}{c}\text { Tempé- } \\
\text { rature } \\
\left({ }^{\circ} \mathrm{C}\right) \\
\\
22,0\end{array}$} & \multicolumn{2}{|c|}{$\begin{array}{c}\text { Direction et } \\
\text { vitesse du vent } \\
(\mathrm{m} / \mathrm{s})\end{array}$} \\
\hline & & & & WNW & 13 & & & SSE & 15 & & & $\mathrm{SE}$ & 12 \\
\hline$\ll$ & $15 \mathrm{~h} 00$ & 831,3 & 7,0 & WNW & 11 & 1002,5 & 20,0 & SSE & 11 & 1000,6 & 21,4 & SE & 10 \\
\hline « & $18 \mathrm{~h} 00$ & $831, \tilde{0}$ & 6,6 & NW & 08 & 1002,3 & 20,3 & $\mathrm{~N}$ & 07 & 1001,1 & 21,7 & $\mathrm{SE}$ & 07 \\
\hline$\ll$ & $21 \ln 00$ & 831,3 & 6,2 & NNW & 07 & 1002,2 & 15,0 & $\mathrm{~N}$ & 07 & 1001,4 & 15,6 & $\mathrm{~N}$ & 07 \\
\hline$\ll$ & $24 \mathrm{~h} 00$ & 830,9 & 5,9 & NNW & 06 & 1001,3 & 15,2 & $\mathrm{~N}$ & 03 & 1001,7 & 15,4 & $\mathrm{~N}$ & 05 \\
\hline $4-10-58$ & $00 \mathrm{hl} 00$ & 838,3 & 9,3 & SSE & 18 & 1013,8 & 19,8 & SSE & 06 & 1013,3 & 20,3 & SE & 04 \\
\hline « & $03 \mathrm{~h} 00$ & 837,8 & 10,5 & $\mathrm{~S}$ & 18 & 1012,1 & 20,0 & $\mathrm{SE}$ & 12 & 1011,7 & 20,3 & $\mathrm{SE}$ & 08 \\
\hline$\ll$ & $06 \mathrm{~h} 00$ & 836,9 & 11,2 & $\mathrm{~S}$ & 15 & 1010,8 & 20,0 & SE & 10 & 1010,7 & 20,5 & SE & 11 \\
\hline$\ll$ & 09 h 00 & 839,1 & 5,8 & W & 11 & 1011,7 & 21,4 & $\mathrm{SE}$ & 13 & 1010,5 & 21,2 & SSE & 13 \\
\hline$\ll$ & 12 h 00 & 839,4 & 6,4 & WNW & 07 & 1013,0 & 14,7 & $\mathrm{~N}$ & 06 & 1012,9 & 15,7 & $\mathrm{~N}$ & 06 \\
\hline
\end{tabular}

B. - HAUTEURS DES PRÉCIPITATIONS (en mm).

\begin{tabular}{|c|c|c|c|c|c|c|c|c|}
\hline \multirow{2}{*}{ Stations } & \multicolumn{2}{|c|}{$29-9-58$} & \multicolumn{2}{|c|}{$30-9-58$} & \multicolumn{2}{|c|}{$1-10-58$} & \multicolumn{2}{|c|}{$4-10-5 s$} \\
\hline & $6 \mathrm{ho0}$ & 18 h 00 & $6 \mathrm{~h} 00$ & $18 \mathrm{~h} 00$ & $6 \mathrm{ho} 00$ & 18 h 00 & $6 \mathrm{hl} 00$ & 18 h 00 \\
\hline Mont Aigoual. & 5 & 38 & 110 & 180 & 19 & 0 & 21 & 52 \\
\hline Nînes ... & 0 & 0 & 0 & 4 & 2 & 0 & 0 & 43 \\
\hline MONTPELLILR . . . & 0 & 0) & 0 & 2 & i & 0 & 3 & 28 \\
\hline
\end{tabular}




\section{I. - Les précipitations}

a) LES TYPES DE PRECIPITATIONS GÉNÉRATRICES DE CRUES DU VIDOURLE.

Considérées du point de vue de la formation d'ondes de crues :

1. Les précipitations d'origine convective (averses orageuses très localisées, de durée assez brève et de forte intensité) ne présentent pas de danger en raison de leur extension limitée dans le temps et l'espace.

2. Les précipitations purement orographiques qui affectent le relief ont généralement leur centre de gravité à l'extérieur du bassin vers le massif de l'Aigoual. Elles peuvent donner des crues assez importantes à l'amont de Sauve qui vont s'amortissant rapidement vers l'aval.

3. Les crues du Vidourle les plus violentes se produisent à partir de précipitations d'origine cyclonique, qui résultent du soulèvement des masses d'air méditerranéen humide et instable par un flux d'air polaire martime descendant le couloir rhodanien. Cette évolution déclenche sur tout le bassin des précipitations intenses, amplifiées par efret orographique, qui se déplacent d'abord dans la direction des vents pluvieux de secteur sud,

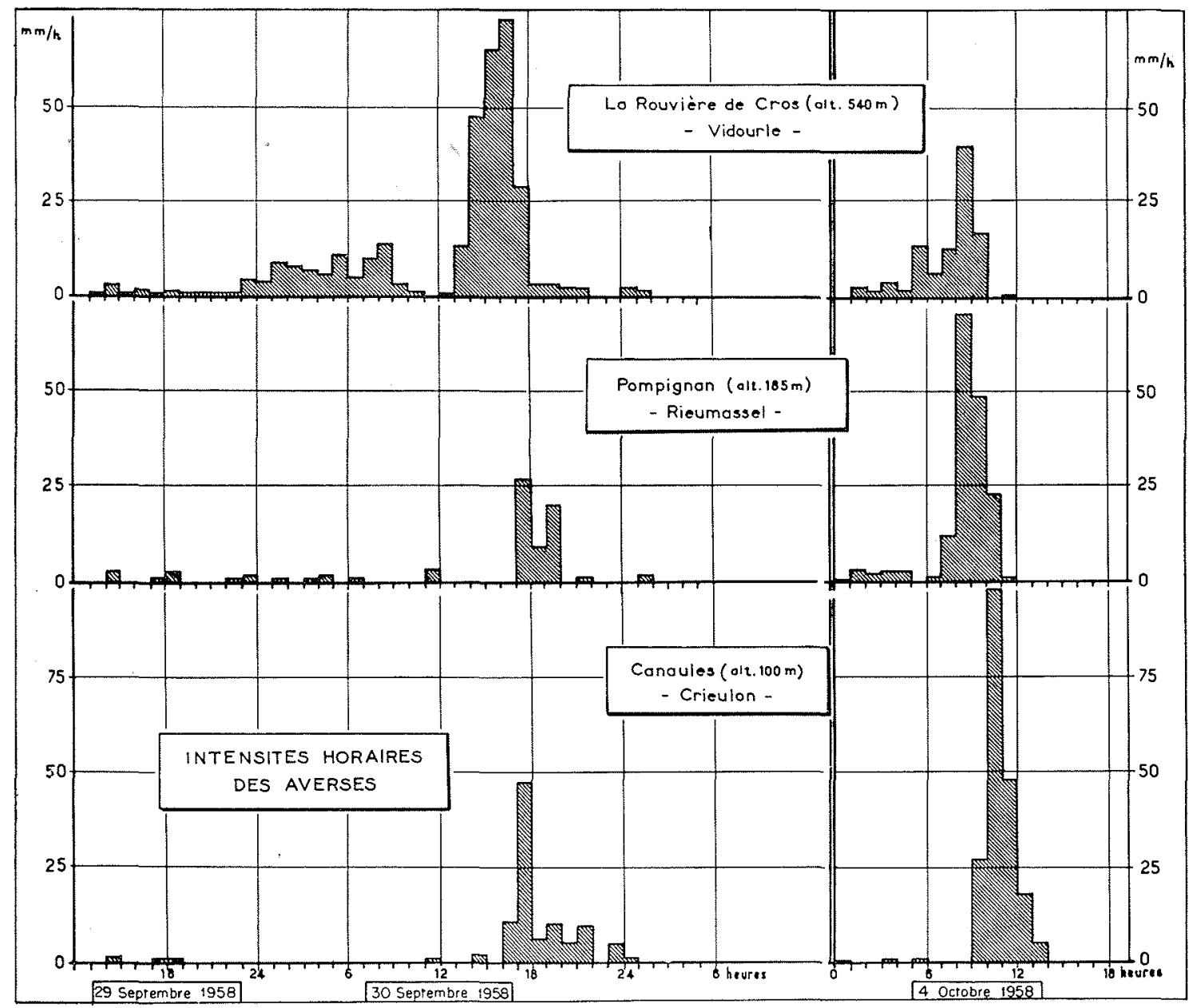

FIG. 1

Intensités horaires des averses des 29-30 septembre et 4 octobre 1958. 
pour redescendre ensuite vers la mer au fur et à mesure de l'avancée du front froid ver's le sud. Cette circonstance est particulièrement importante pour expliquer le caractère subit et violent des crues du Vidourle et de ses affluents: Le sens de déplacement des averses de l'amont vers l'aval tend à renforcer et raidir le front d'onde de crue au fur et à mesure de sa propagation. Les précipitations qui ont causé les crues des 30 septembre et 4 octobre 1958 appartiennent à cette classe.

Le processus décrit apparaît avec une particulière netteté lorsque l'on rapproche du tableau I, groupant les observations météorologiques des postes de l'Aigoual, Nìmes, Montpellier et sur lequel ont été repérés les passages de fronts froids (rotation des vents, baisse de température, hausse de pression), les diagrammes d'intensités horaires des averses enregistrées sur le bassin du Vidourle les 30 septembre et 4 octobre 1958 (fig. 1).

b) LA DISTRIBUTION SPATIALE DES AVERSES GÉNÉRATRICES DE CRUES.

Les crues produites par ces aver'ses cycloniques présentent des caractéristiques très diverses selon la répartition spatiale des précipitations sur le bassin. On peut distinguer les dominantes ci-après :

- Des crues importantes dès le haut-bassin, mais qui ne s'aggravent pas à l'aval : le centre de gravité des précipitations se trouve sur les Cévennes méridionales à l'extérieur du bassin (cas de la crue du 30 septembre 1958 : (cf. carte $\mathrm{n}^{\prime \prime 2}$ ).

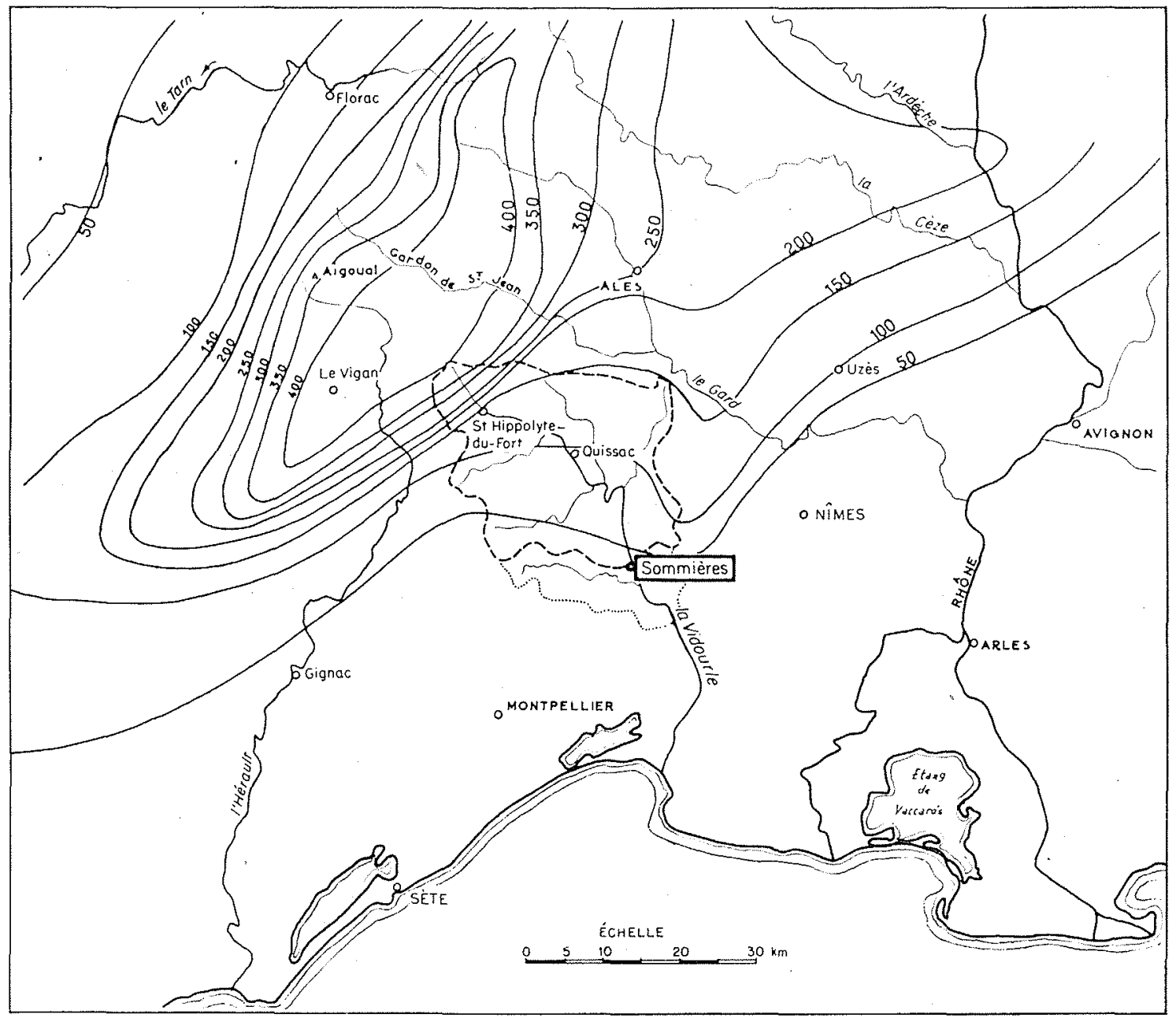

Carte No 2. - Crues du Vidourle de l'automne 1958.

Isohyètes, cotées en mm de lame d'eau, des précipitations cumulées des 29 et 30 septembre 1958 (de 0 à 24 h). 


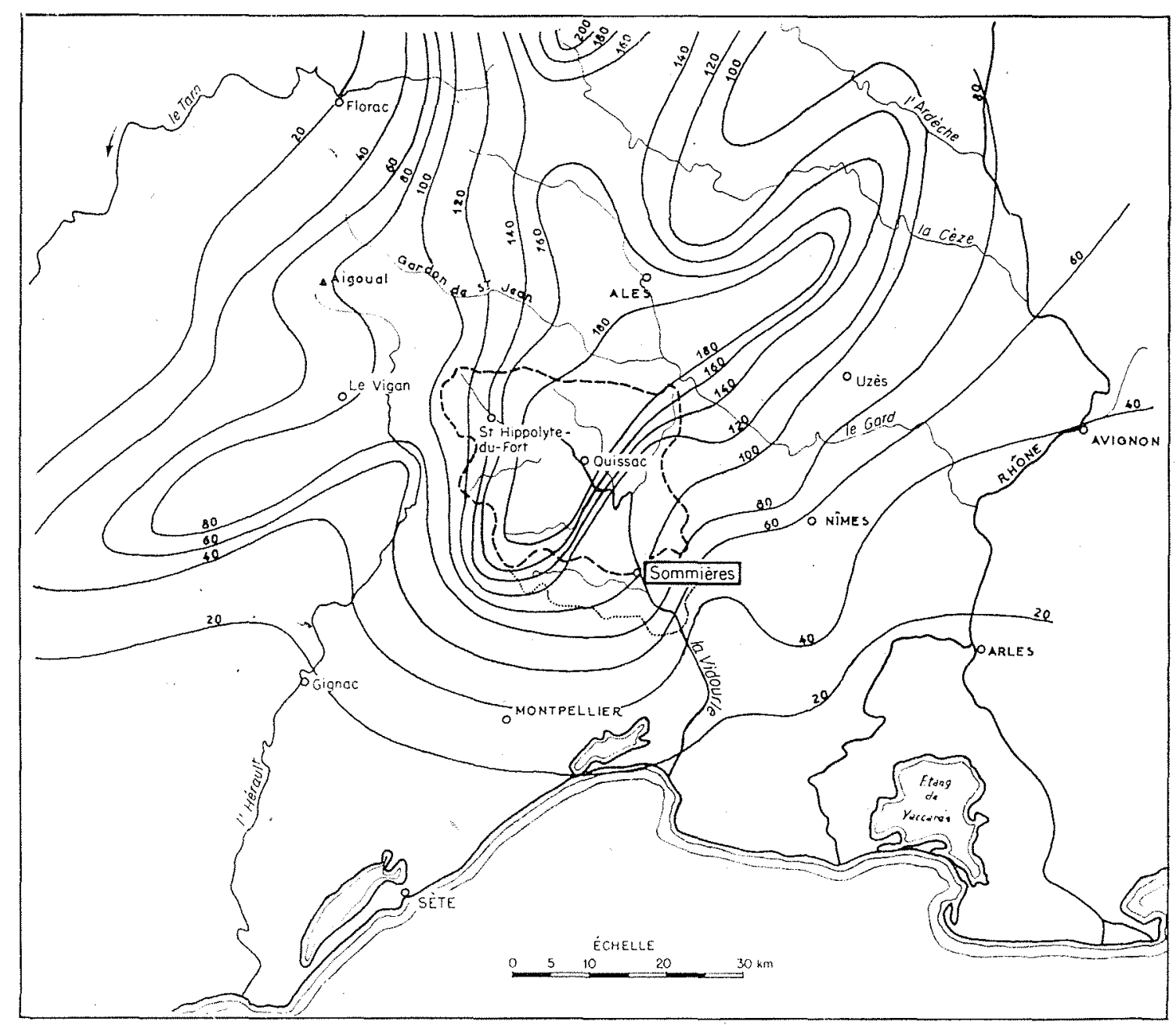

Carte No 3. - Crues du Vidourle de lautomne 1958.

Isohyètes, cotées en mm de lame d'eau, des précipitations du 4 octobre 1958 (de 0 à $24 \mathrm{~h}$ ).

- Des crues importantes sur le haut-bassin et qui vont s'exacerbant vers l'aval : les précipitations sont centrées sur la partie médiane du bassin (cas de la crue du 4 octobre 1958 : cf. carte $\left.n^{\circ} 3\right)$.

- Des crues qui se forment à l'aval de Sauve : le centre de gravité des précipitations situé sur la partie orientale du bassin exerce une action conjuguée sur les affluents de rive gauche du Vidourle, Crieulon, Courme d'une part, et sur le Vistre d'autre part. Les crues qui en résultent sont particulièrement dangereuses à Sommières et à l'aval dans la traversée de la plaine du Bas-Languedoc.

Un déplacement de $10 \dot{a} 20 \mathrm{~km}$ du centre de gravité des averses exerce nne influence determinante sur la physionomie des crues et constitue l'un des obstacles majeurs à la prévision ou l'annonce des crues du Vidourle. c) L'ANALYSE DES INTENSITÉs ET DURÉEs d'AVERSES.

La rapidité d'évolution du phénomène «aversecrue » nécessite - outre un réseau assez dense de pluviomètres permettant de déterminer avec suffisamment de prévision la répartition spatiale des précipitations (cf. supra $b$ ) - l'utilisation de pluviographes pour étudier l'intensité et la durée des averses, parametres indispensables à la recherche d'une relation pluie-débit.

L'analyse de la structure des averses génératrices de crues d'après les pluviographes du bassin a donné les résultats suivants:

1. La répartition dans le temps des intensités d'averses orageuses isolées présente génèralement au maximum au début de l'averse, mais la phase la plus violente des pluies cycloniques, qui accompagne par exemple la descente d'un front froid, survient après des 
précipitations moins intenses qui ont pu saturer au moins partiellement le bassin. L'averse qui a donné $230 \mathrm{~mm}$ en $4 \mathrm{~h}$ le 30 septembre 1958 à la Rouvière-de-Cros avait été précédée de $100 \mathrm{~mm}$ de précipitations en 24 heures (cf. fig. 1).

2. La recherche des intensités maxima de précipitations pour des durées de référence déterminées depuis $15 \mathrm{mn}$ jusqu'à plus de 30 heures, a montré que le record était détenu par l'averse du 30 septembre 1958 sur le haut bassin (pluviographe de la Rouvière-de-Cros) et par l'averse du 4 octobre au centre du bassin (pluviographes de Pompignan et de Canaules) avec près de $100 \mathrm{~mm}$ en 1 heure.

L'établissement de graphiques intensitédurée-fréquence d'averse est évidemment prématuré après 2 années d'exploitation seule- ment. Néanmoins, la comparaison avec les intensités maxima d'averses enregistrées à Montpellier (Bel-Air) au cours de la période 1919-1949, laisse présumer que les intensités atteintes au cours des averses du 30 septembre 1958 correspondent à des fréquences ou probabilités d'occurrence assez faibles (cf. fig. 2).

\section{II. - L'incidence des caractéristiques thermiques du bassin sur les crues}

Cette incidence est perceptible en été principalement où les averses orageuses de juilletaoût, intenses et de brève durée, sont neutralisées par la recharge en eau du bassin soumis à une évaporation très active.

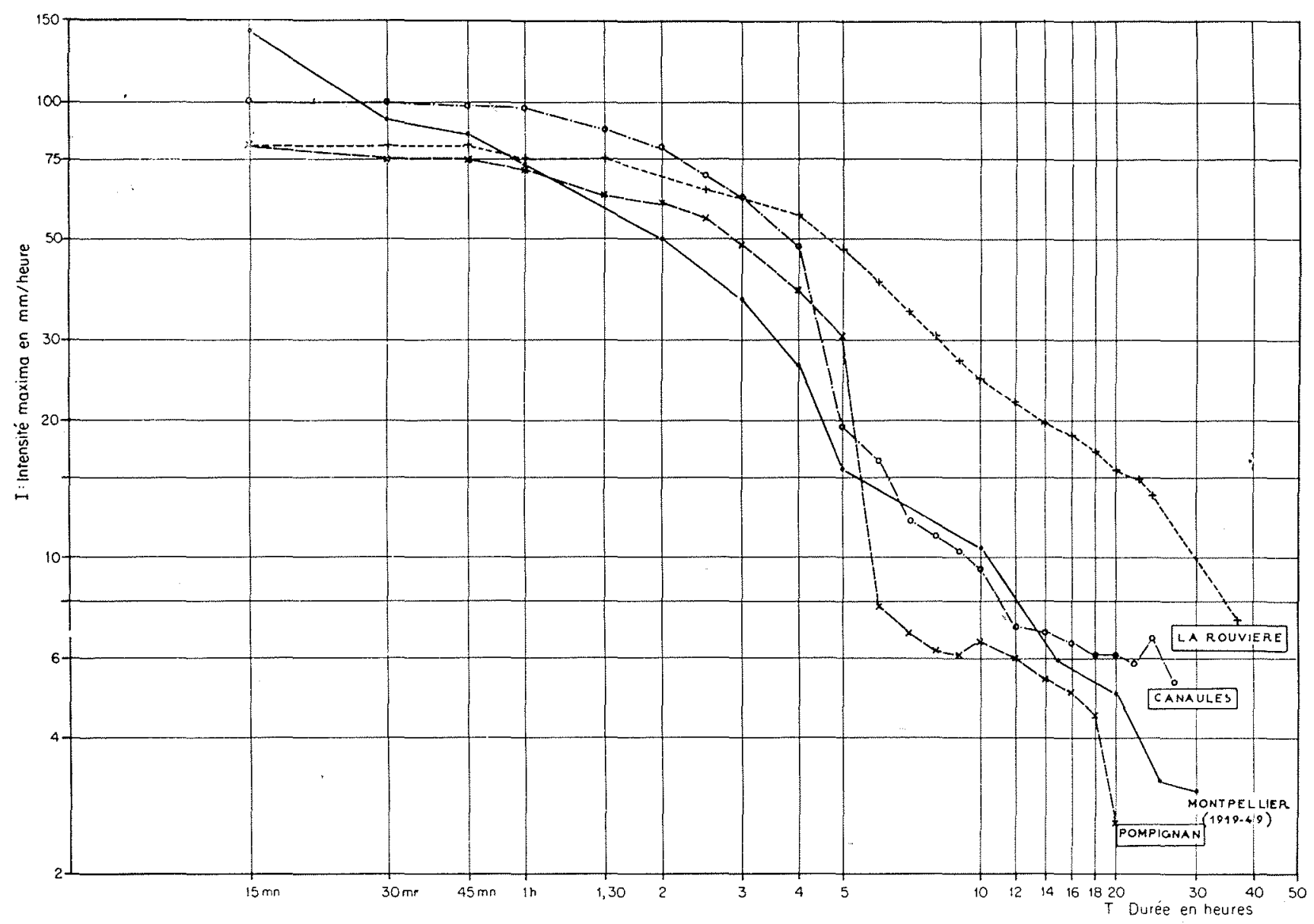

Fic. 2

Courbes intensité-durée $\log I=f(\log T)$ des averses enregistrées sur le bassin du Vidourle (1956-1958). 
L'importance de l'évaporation apparait principalement dans la rapidité de tarissement du bassin dont les crues constituent l'essentiel de l'écoulement.

\section{C. - LES OBSERVATIONS DE NIVEAUX ET MESURES DE DÉBITS DE CRUE}

\section{I. - Niveaux atteints par les crues}

a) RÉPARTITION DES POINTS D'OBSERvation (cf. carte $n^{\circ} 1$ ).

b) ANalyse des obSERvations LimnimétriQues ANCIENNES : RÉPARTITION DES CRUES DU Vidovrle a Sommières.

Une analyse statistique des cotes maxima observées au pont de Sommières de 1890 à 1957 donne les résultats suivants concernant la répartition des crues.

\section{Dans le temps :}

$46 \%$ des crues maxima annuelles se produisent en septembre-octobre-novembre;
$29 \%$ des crues maxima annuelles se produisent en décembre-janvier-février;

$16 \%$ des crues maxima annuelles se produisent en mars-avril-mai;

$9 \%$ des crues maxima annuelles se produisent en juin-juillet-août.

\section{Par importance:}

La crue annuelle de fréquence moyenne :

$10 \%$ (crue décennale) correspond à la cote $5,40 \mathrm{~m}$;

$20 \%$ (crue quinquennale) correspond à la cote $4,70 \mathrm{~m}$;

$50 \%$ (crue biennale) correspond à la cote $3,10 \mathrm{~m}$ : à cette cote, la ville de Sommières est déjà atteinte par l’inondation.

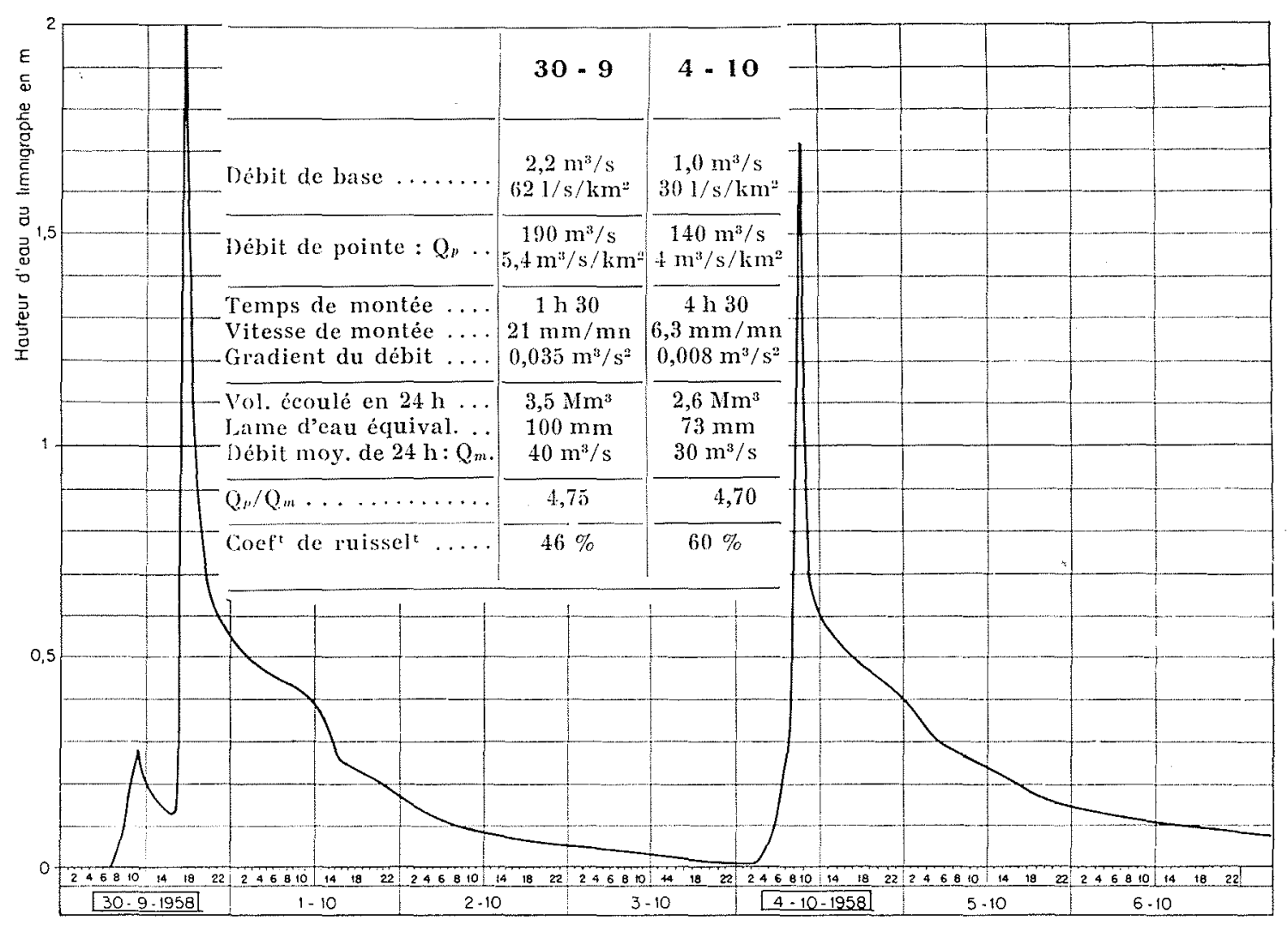

FIG. 3

Enregistrement des crues du Vidourle à Saint-Hippolyte-du-Fort (bassin versant : $35,5 \mathrm{~km}^{*}$ ). 


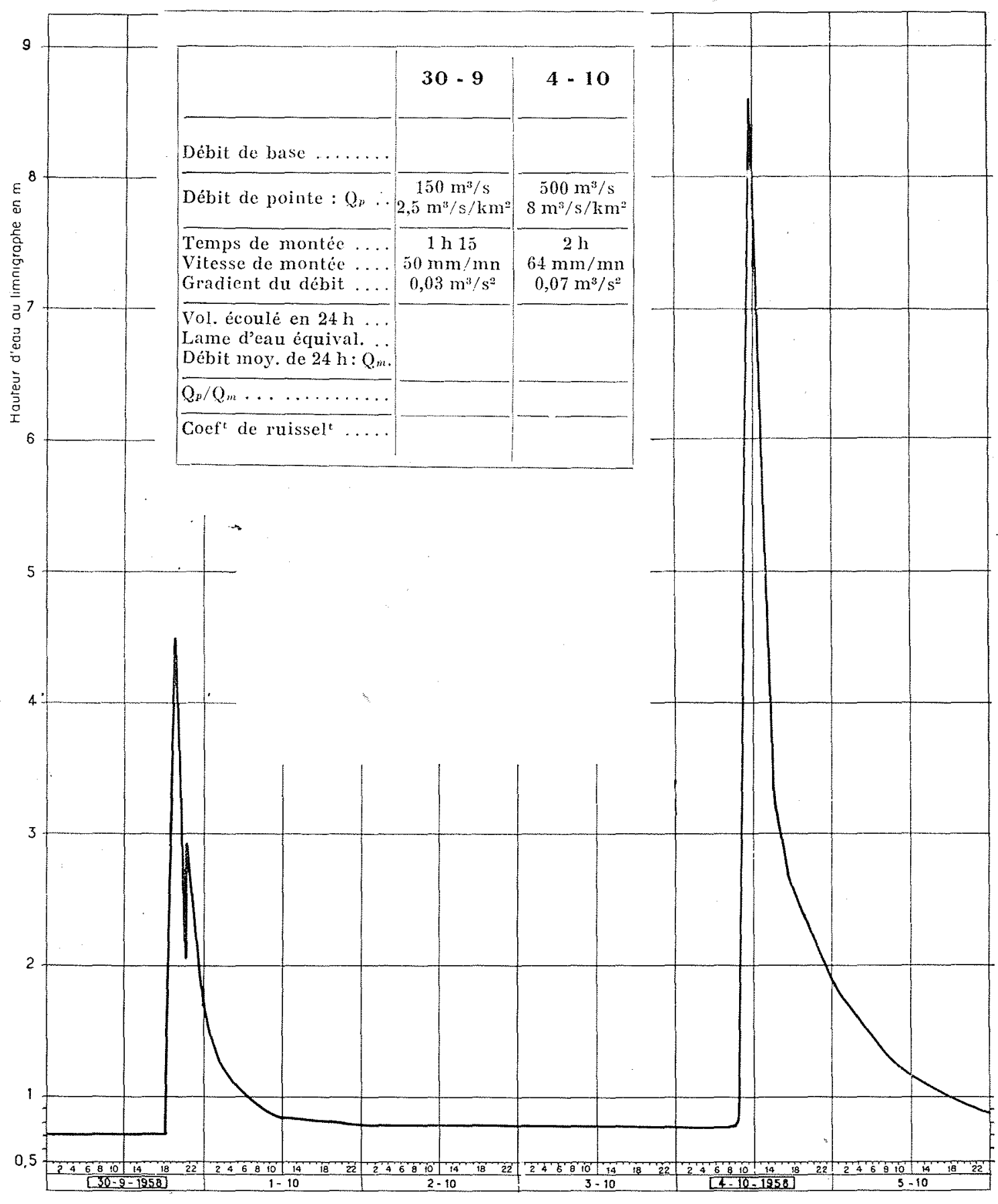

Firs. 4

Enregistrement des crues du Rieumassel au pont de Tarrieu (bassin versant : $60 \mathrm{~km}^{2}$ ).

3. Dans le temps et par importance: on constate une concentration croissant avec l'importance des crues dans les mois d'automne où se produisent $35 \%$ des crues observées, $55 \%$ des crues dépassant $3 \mathrm{~m}$ et $90 \%$ des crues dépassant $5 \mathrm{~m}$, le phénomène inverse ayant lieu au printemps. c) Comparaison des niveaux atTeints PaR Les CRUES DE 1958 AUX RECORDS DE CRUES ANCIENNES SUR LE BASSIN DU VidouRle.

Des crues des $1^{\mathrm{er}}$ et 4 octobre 1958 , dont nous reproduisons les hydrogrammes enregistrés sur le bassin (cf. fig. 3 à 6 ), seule la crue du 4 octobre est comparable, du point de vue des niveaux. 
T A B L E A U II

COMPARAISON DES COTES MAXIMA (en m) ATTEINTES PAR LES CRUES AUX ÉCHELLES LIMNIMETRIQUES DU VIDOURLE ET DE SES AFFLUENTS

\begin{tabular}{|c|c|c|c|c|c|c|c|c|c|c|c|}
\hline Date des crues & 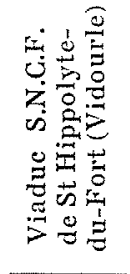 & 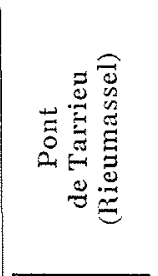 & 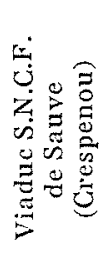 & 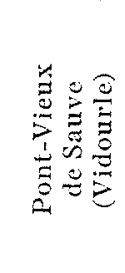 & 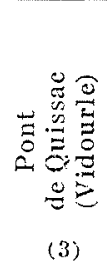 & 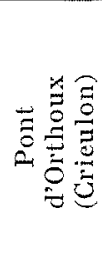 & 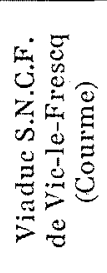 & 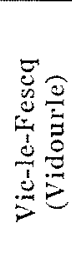 & 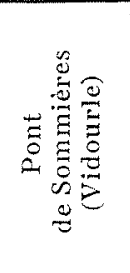 & 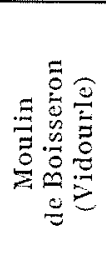 & 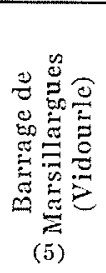 \\
\hline $30-9,1-101958$ & 2,85 & 4,47 & 2,60 & 3,80 & 4,60 & 2,98 & 1,80 & 3,60 & 2,10 & 2,70 & 1,80 \\
\hline $4-10,1958 \ldots$ & 2,10 & 8,00 & 5,00 & () 7,00 & 7,00 & 7,20 & 7,00 & 7,60 & $\begin{array}{l}\text { (4) } 6,76 \\
\text { au limni- } \\
\text { graphe }\end{array}$ & 6,50 & 4,54 \\
\hline $\begin{array}{r}1-10,1723 \ldots \\
17-9,1858 \ldots\end{array}$ & & . & & 8,30 & $\begin{array}{l}8,40 \\
6,74\end{array}$ & 7,87 & & & $\begin{array}{l}(>7,70) \\
(>7,00)\end{array}$ & 6,60 & 4,15 \\
\hline $27-9,1933 \ldots$ & $(>2,00)$ & (1) $(11 ; 00)$ & & 9,00 & 8,05 & & & & $\begin{array}{c}7,70 \\
\text { repere } \\
\text { au } \\
\text { droit } \\
\text { du pont }\end{array}$ & & 4,57 \\
\hline
\end{tabular}

(1) Coté estimée après coup.

(2) La crue du 4 octobre 1958 aurait dépassé largement $7 \mathrm{~m}$ au Pont-Vieux de Sauve sans les travaux de calibrage du lit exécutés en 1957.

(3) La comparaison des maxima de crues est rendue délicate par l'instabilité du lit au pont de Quissac.

(4) La cote $6,76 \mathrm{~m}$ est celle enregistrée au limnigraphe fixé contre la face aval du pont, côté R.G. Un nivellement effectué immédiatement après la crue a donné les résultats suivants :

- Cote NGF des P.H.E. $40 \mathrm{~m}$ à l'amont du pont : 28,64 , soit $7,67 \mathrm{~m}$ à l'échelle.

- Cote NGF des P.H.E. $40 \mathrm{~m}$ à l'aval du pont : 27,89 , soit $6,92 \mathrm{~m}$ à l'échelle.

On peut ainsi évaluer le remous dû à l'ouvrage $(0,75 \mathrm{~m})$ et constater que la crue de 1958 a égalé l'importance des plus grandes crues observées à Sommières.

(5) Ces cotes ne peuvent servir à des comparaisons très précises en raison des déversements et ruptures de digues qui se produisent généralement à l'amont ou à l'aval du barrage de Marsillargues lors dies crues importantes.

maxima atteints, aux records du passé détenus par les crues des $1^{\text {er }}$ octobre 1723,17 septembre 1858 et 27 septembre 1933 (cf. tableau II).

Il ressort de cette comparaison que la crue du 4 octobre 1958 :

- n'a pas atteint l'importance de la crue de 1933 à l'amont de Quissac;

- a atteint sinon dépassé l'importance de la crue de 1933 entre Vic-le-Fesq et Sommières (certains repères de la crue de 1933 ont été dépassés de 0,30 à $0,40 \mathrm{~m}$ ) au débouché de la zone découverte par l'isohyète maximum de l'averse (cf. carte $\mathrm{n}^{\circ} 3$ );

- n'a pas dépassé les niveaux de la crue de 1933 à l'aval de Sommières.

\section{1. - Les mesures et estimations des débits de crues}

a) JAUGEAGES ET ESTIMATIONS DE DÉBITS DE CRUES ANCIENNES.

Le Vidourle n'a fait l'objet d'aucun jaugeage de crue avant 1956. Au cours d'une campagne de mesures (1956-1957), un étalonnage sommaire a pu être réalisé pour les stations suivantes :

Saint-Hippolyte-du-Fort (courbe de tarage calculée pour seuil épais)

Sauve $\left(400 \mathrm{l} / \mathrm{s} / \mathrm{km}^{2}\right.$ mesurés)

Orthoux $\left(600 \mathrm{l} / \mathrm{s} / \mathrm{km}^{2}\right.$ mesurés $)$

Sommières $\left(600 \mathrm{l} / \mathrm{s} / \mathrm{km}^{2}\right.$ mesurés)

Un premier essai de détermination des ordres 
T A B L E A U III

COMPARAISON DES DÉBITS DE CRUES DU VIDOURLE ET DE SES AFFLUENTS

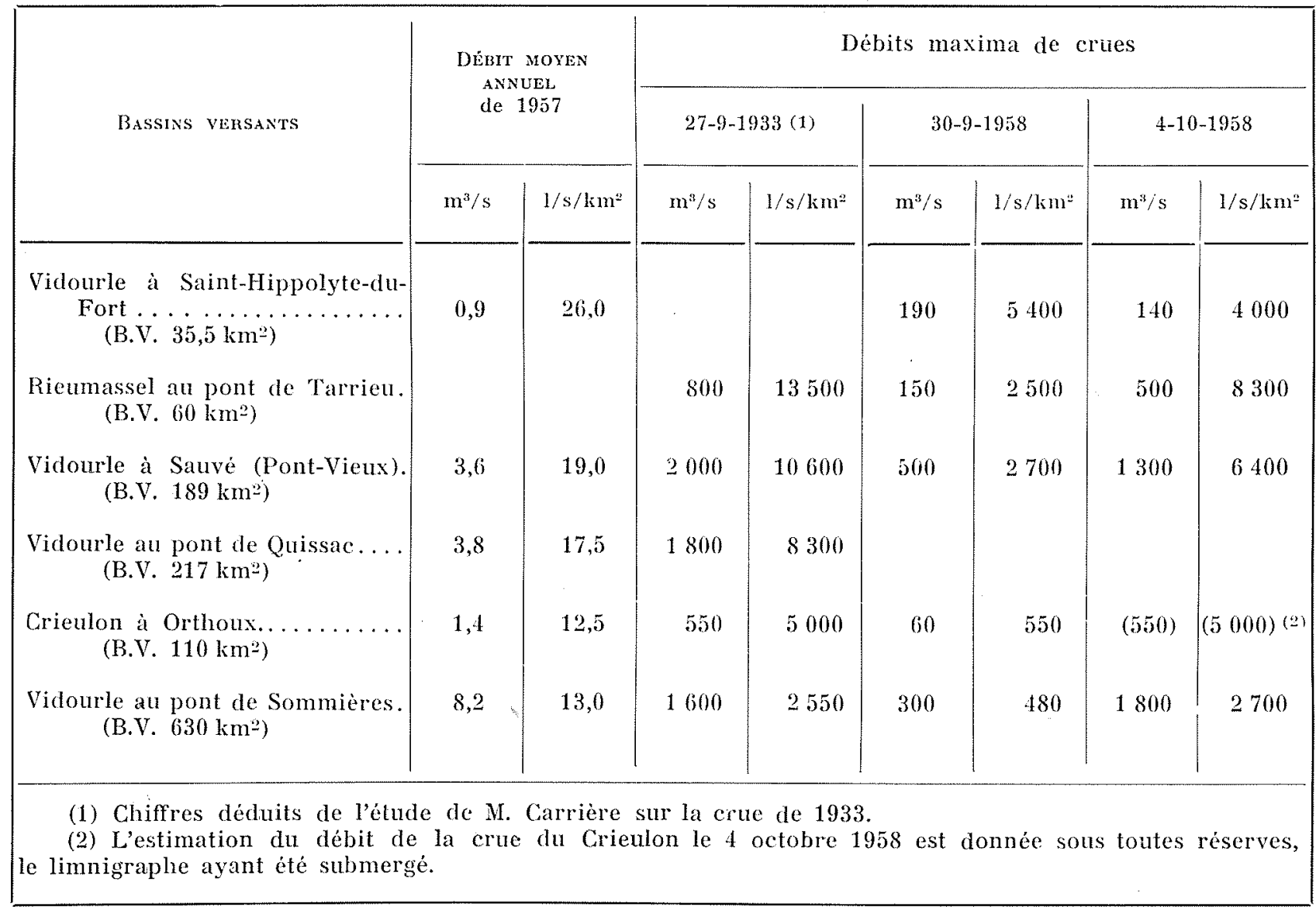

de grandeur des débits spécifiques maxima de crues a été effectué en 1938, sur diverses sections des cours du Vidourle et de ses affluents, par M. Carrière, ingénieur des Ponts \& Chaussées à Nìmes, d'après les relevés de traces des plus hautes eaux de la crue de 1933.

\section{b) DÉBits des crues de 1958.}

L'estimation des débits de pointe de la crue du 4 octobre - ceux de la crue du $1^{\text {er }}$ ont pu être déterminés à l'aide des courbes de tarage des stations - a fait l'objet de divers essais afin de recouper les chiffres obtenus en utilisant les observations de remous au franchissement de singularités (ponts, seuils, etc.) et des relevés de profils de ligne d'eau sur un certain nombre de sections du Vidourle et de ses affluents. Les chiffres retenus représentent des ordres de grandeur vraisemblables pour les débits maxima instantanés (cf. tableau III). Leur examen conduit aux constatations suivantes :

1. Les débits de pointe peuvent atteindre et dé- passer 200 fois la valeur du module sur le bassin du Vidourle (comparaison des crues de 1933 et 1958 aux débits moyens de 1957).

2.Il semble que les débits spécifiques maxima observés le 4 octobre se soient produits sur le Crespenou, affluent de rive gauche débouchant à Sauve, où l'on a estimé à $11 \mathrm{~m}^{3} / \mathrm{s} / \mathrm{km}^{2}$ la crue d'un bassin versant de $36 \mathrm{~km}$.

3. La protection totale de la ville de Sommières, réalisée par écrétement de la crue du 4 octobre à $400 \mathrm{~m}^{3} / \mathrm{s}$, aurait exigé le stockage de $24 \mathrm{M} \mathrm{m}^{3}$ à l'amont.

4. Le débit maximum ayant transité dans le lit endigué du Bas-Vidourle est évalué à $850 \mathrm{~m}^{3} /$ seconde $\left(1 \mathrm{~m}^{3} / \mathrm{s} / \mathrm{km}^{2}\right)$ au barrage de Marsillargues : il se produit une atténuation importante $(50 \%)$ de l'onde de crue à l'aval de Sommières. Ce phénomène est dû aux effets conjugués du stockage, des déversements et de la propagation de la crue dans un chenal à très faible pente. 


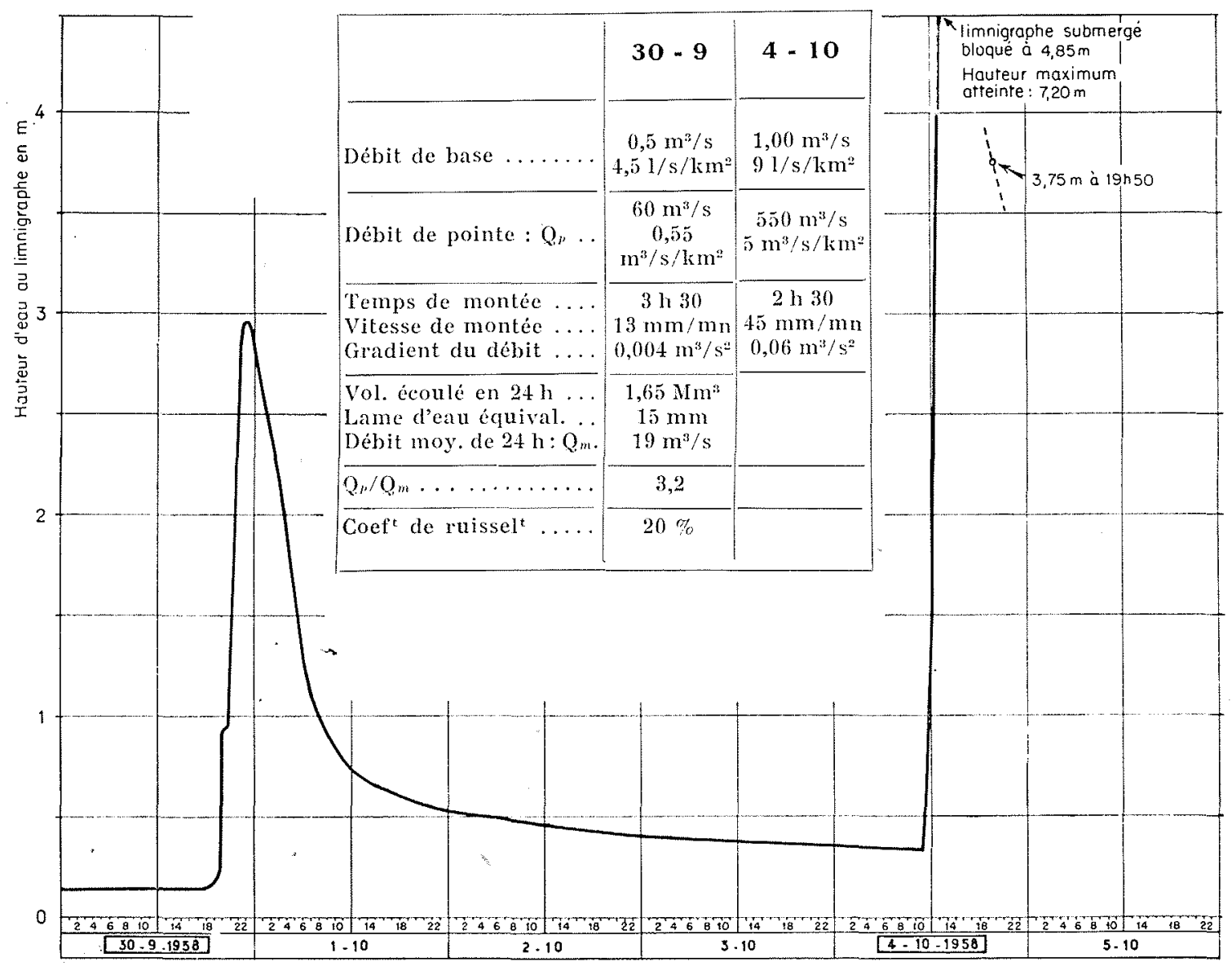

Fig. 5

Enregistrement des crues du Crieulon à Orthoux (bassin versant : $110 \mathrm{~km}$ ) .

\section{III. - Evolution et propagation des crues}

La rapidité extrême d'évolution des crues du Vidourle est mise en évidence par les chiffres suivants :

a) Les vitesses de montée du plan d'eau à plus de $50 \mathrm{~mm} / \mathrm{mn}$ déduites des limnigrammes ont été dépassés le 4 octobre au pont de Quissac, où les eaux sont passées de la cote $2,50 \mathrm{~m}$ à la cote $7,00 \mathrm{~m}$ en 45 minutes, soit à la vitesse extraordinaire de $100 \mathrm{~mm} / \mathrm{mn}$. Ces chiffres sont significatifs du degré de «non-permanence » de l'écoulement des crues du Vidourle, et, corrélativement, de l'impossibilité pratique de les jauger directement.

b) Les temps de propagation des crues entre Saint-Hippolyte-du-Fort et Sommières $(47 \mathrm{~km})$ varient de $9 \mathrm{~h}$ le 1 er octobre à $6 \mathrm{~h} .30$ le 4 , ce qui correspond à des vitesses moyennes de 1,45 et $2,00 \mathrm{~m} / \mathrm{s}$. Ces temps et vitesses ne sont pas uni- formes sur tout le parcours : on observe des vitesses dépassant $3 \mathrm{~m} / \mathrm{s}$ dans des sections encaissées. On retiendra que les crues du Vidourle atteignent Aigues-Mortes 12 à $16 \mathrm{~h}$ après SaintHippolyte-du-Fort.

c) Le retard de la crue sur l'averse génératrice varie selon les types de crues, la direction et la vitesse du déplacement de l'averse. Les chiffres suivants peuvent être admis comme valeurs moyennes :

2 à $3 \mathrm{~h}$ pour le Vidourle à Saint-Hippolyte-duFort.

$7 \mathrm{~h}$ pour le Crieulon à Orthoux $(3 \mathrm{~h}$ le 4-10-1958).

$10 \mathrm{~h}$ pour le Vidourle à Sommières $(6 \mathrm{~h}$ le 4-10-1958).

La brièveté de cet intervalle de temps rend très difficile l'annonce des crues sur le bassin du Vidourle, ou les temps de propagation sont également courts. 


\section{D. - L'APPLICATION DE LA MÉTHODE DE L'HYDROGRAMME UNITAIRE AUX CRUES DU VIDOURLE}

La rapidité d'évolution et la violence de ces phénomènes conduit à rechercher un procédé d'évaluation du débit de pointe afférent à une averse de durée et d'intensité données. En effet, la brièveté des averses «efficaces» et la variabilité du rapport débit de pointe/débit de $24 \mathrm{~h}$ (calculé à partir du début de la crue) ne permettent pas de juger de l'importance relative des crues à partir des précipitations et débits moyens journaliers. Par contre, les débits de pointe étant dus essentiellement aux apports du. ruissellement superficiel, l'utilisation de la méthode de l'hydrogramme unitaire, qui permet d'étudier précisément l'hydrogramme de cette phase d'écoulement de crue, parait tout indiquée ici.

En bref', la théorie de l'hydrogramme unitaire se réduit aux principes suivants :

I. Le temps de concentration (durée de trajet maximum accompli par l'eau de ruissellement pour atteindre l'exutoirè) est une grandeur supposée constante pour un bassin donné. On peut en déterminer expérimentalement la valeur en étudiant sur des hydrogrammes de crues les durées d'écoulement des apports du ruissellement superficiel dont on retranche la durée de l'averse productrice de ce ruissellement. On retiendra comme ordre de grandeur des temps de concentration :

6 à $8 \mathrm{~h}$ sur le bassin du Vidourle à SaintHippolyte-du-Fort,

12 à $14 \mathrm{~h}$ sur le bassin du Crieulon à Orthoux,

16 à $18 \mathrm{~h}$ sur le bassin du Vidourle à Sommières.

II. Tous les hydrogrammes de ruissellement de crues produites par des averses de même durée, suffisamment brèves par rapport an temps de concentration du bassin étudié, sont des courbes affines dont les ordonnées sont proportionnelles aux volumes de ruissellement mis' en ouvre, La durée «efficace» (du point de vue de la production du ruissellement) des averses du $30-9$ et du 4-10-1958 a varié de 1 à $3 \mathrm{~h}$, ce qui permet de les considérer comme des averses unitaires.

III. L'hydrogramme résultant d'une averse complexe non unitaire s'obtient en représen- tant celle-ci par une succession d'averses unitaires et en composant par sommation d'ordonnées les hydrogrammes - convenablement décalés dans le temps afférents à ces averses élémentaires.

On détermine pour chaque bassin considéré :

- L'importance de la phase du ruissellement superficiel selon les crues : étude du coefficient de ruissellement.

- La distribution dans le temps de l'écoulement de cette phase : étude de la forme de l'hydrogramme de ruissellement d'une averse unitaire de durée déterminée et plus spécialement détermination de la valeur du débit de pointe correspondant à un volume de ruis. sellement donné.

a) Les valeurs du coefficient de ruissellement, défini par le rapport du volume ruisselé au volume précipilé sur le bassin au cours de l'averse «efficace» ayant provoqué la crue, ne paraissent pas devoir dépasser $60 \dot{a} 70 \%$ et sont très dispersées pour toutes les crues étudiées. Ce fait vaut d'être noté car il ne semble pas que l'on puisse atteindre des valeurs voisines de $100 \%$ même lors des qrues «catastrophiques». Le facteur «pertes» de l'averse (rétention par infiltration, même limitée aux couches superficielles du sol, qui distrait momentanément une frac tion du débit pluvial, etc...) est important et a dépassé $20 \mathrm{~mm} / \mathrm{h}$ en moyenne pendant les averses efficaces des crues de 1958 .

b) Des formes d'hydrogrammes de ruissellement correspondant à un volume de ruissellement unité ont été dégagées des hydrogrammes de crues enregistrés sur le Vidourle à SaintHippolyte-du-Fort, à Sommières, et sur le Crieulon à Orthoux pour différentes durées d'averses unitaires. Les débits spécifiques de pointe des hydrogrammes unitaires, correspondant a un volume de ruissellement de $1000 \mathrm{~m}^{3} / \mathrm{km}^{2}$ (1 $\mathrm{mm}$ de lame d'eau) et à une durée d'averse efficace de $1 \mathrm{~h}$, sont les suivants :

$\mathrm{Q}_{\mathrm{I}}=75 \mathrm{l} / \mathrm{s} / \mathrm{km}^{2}$ pour le Vidourle à Saint-Hippolyte-du-Fort.

$\mathrm{Q}_{0}=55 \mathrm{l} / \mathrm{s} / \mathrm{km}^{2}$ pour le Crieulon a Orthoux.

$\mathrm{Q}_{\mathrm{s}}=50 \mathrm{l} / \mathrm{s} / \mathrm{km}^{2}$ pour le Vidourle à Sommières.

Connaissant la lame d'eau précipitée par l'averse «efficace» et le coefficient de ruissellement correspondant, on pourra évaluer le débit de pointe de l'hydrogramme du ruissellement. 


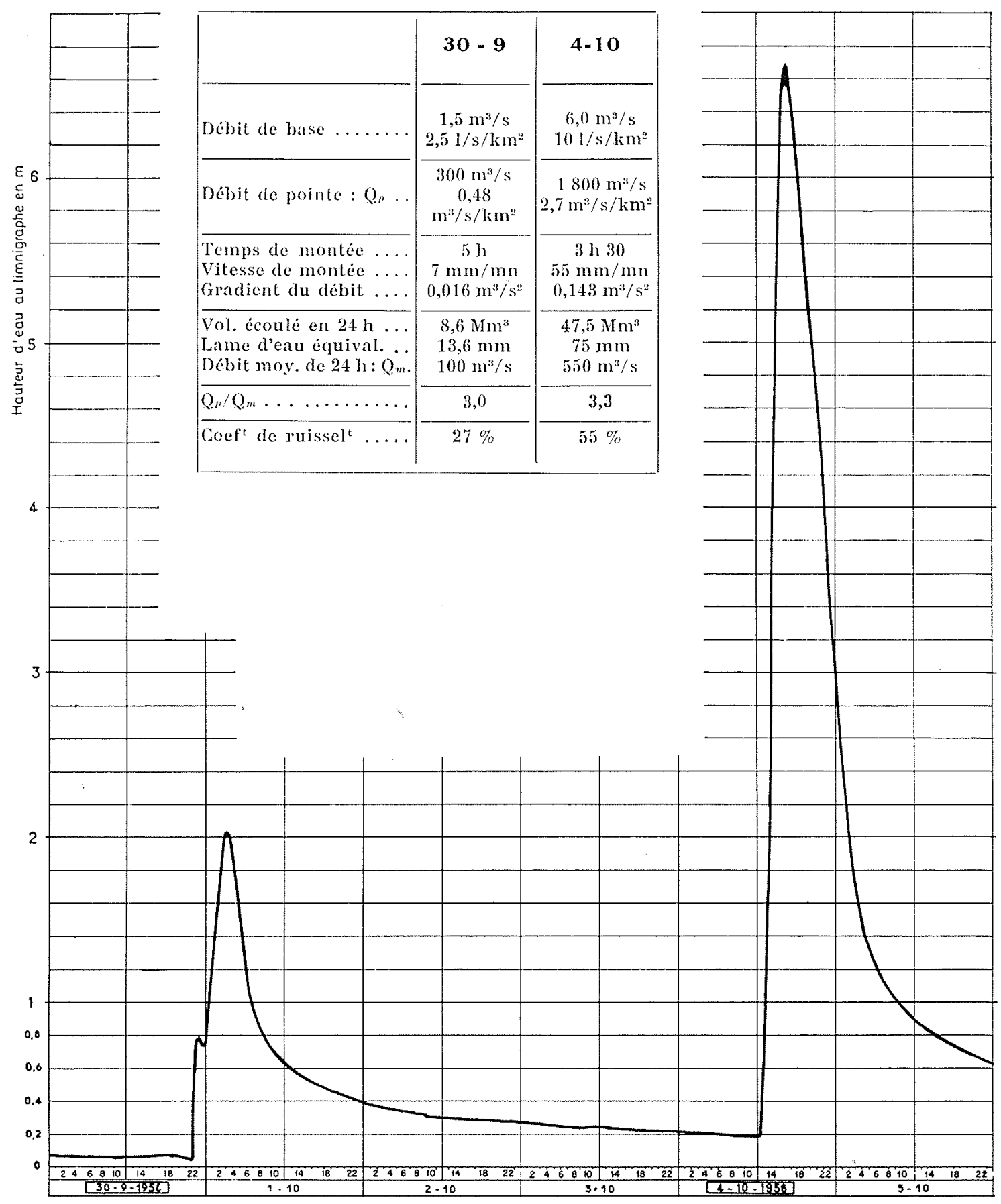

Fic. 6

Enregistrement des crues du Vidourle à Sommières (bassin versant : $630 \mathrm{~km}^{2}$ ).

Le débit de pointe réel, qui prend en compte le débit de base (alimenté par les apports de l'écoulement souterrain), n'est pas sensiblement différent du débit de pointe de ruissellement pour les crues importantes. Le véritable problème revient donc $\dot{a}$ «choisir 》le coefficient de ruis- sellement convenable : s'il se confirme que les valeurs de ce paramètre plafonnent entre 60 et $70 \%$, il devient possible de calculer les ordres de grandeur de débits de crues maxima correspondant aux caractéristiques d'averses définies par des courbes intensité-durée-fréquence. 


\section{CONCLUSION}

Les résultats présentés tendent à montrer que l'application des techniques d'hydrogrammes unitaires à des bassins de plusieurs centaines de $\mathrm{km}^{2}$ est possible. Il y aurait donc intérêt à étendre leur application aux bassins voisins de ceux du Vidourle, soumis aux mêmes types de crues, partout où l'on dispose d'enregistrements simultanés d'averses et des crues subséquentes, afin d'en déterminer les caractéristiques (temps de concentration, etc...). Une phase ultérieure de recherches consistant à relier ces grandeurs aux caractéristiques physiques des bassins, il deviendrait alors possible, à partir d'un réseau un peu plus étoffé en limnigraphes et pluviographes, de prospecter systématiquement la zone névralgique du rebord sud-oriental des Cévennes, de rechercher les points de concentration des crues, et de calculer des hydrogrammes-types sans qu'il soit absolument nécessaire de disposer d'enregistrements limnigraphiques. Une telle information ne serait sans doute pas dépourvue d'intérêt pour les études de protection contre ces crues.

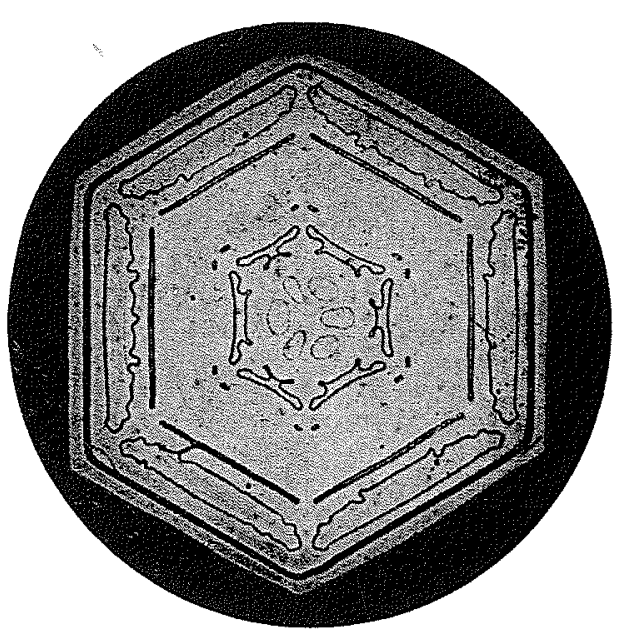

